\title{
The importance of bonding in intralayer carbon fibre/self-reinforced polypropylene hybrid composites
}

\author{
Yentl Swolfs ${ }^{* 1}$, Jia Shi ${ }^{1}$, Yannick Meerten ${ }^{1}$, Peter Hine ${ }^{2}$, Ian Ward ${ }^{2}$, Ignaas Verpoest ${ }^{1}$, Larissa \\ Gorbatikh $^{1}$
}

${ }^{1}$ Department of Materials Engineering, KU Leuven, Kasteelpark Arenberg 44 bus 2450, Belgium

${ }^{2}$ Soft Matter Group, University of Leeds, LS2 9JT, United Kingdom

*Corresponding author: Y. Swolfs (yentl.swolfs@mtm.kuleuven.be)

\begin{abstract}
Polymer composites are usually either stiff or tough, but seldom both. Intralayer hybrids of carbon fibre and self-reinforced polypropylene (PP) do offer the potential to achieve a unique combination of toughness and stiffness. In these hybrids, the bonding between carbon fibre prepregs and PP tapes is a crucial parameter. For a weak bonding, the $20 \%$ ultimate tensile failure strain and high penetration impact resistance of self-reinforced PP were maintained. For a strong bonding, the ultimate tensile failure strain was strongly reduced, but the flexural performance was improved. For a homopolymer PP matrix in the prepregs, the weak bonding between fibre and matrix caused the penetration impact resistance to reduce according to a linear rule-of-mixtures. For a maleic anhydride modified PP matrix however, the strong fibre-matrix bonding greatly reduced the penetration impact resistance. These results provide new insights into designing hybrid composites with a unique balance of stiffness and failure strain.
\end{abstract}

Keywords: A. Carbon fibre; A. Hybrid; A. Polymer (textile) fibre; Self-reinforced composites

\section{Introduction}

Carbon fibre (CF) composites combine excellent stiffness and strength with a low density, making them a preferred choice in many lightweight structural applications. However, they often lack toughness due to a low failure strain. One solution to improve the toughness is to find alternative fibres with a larger failure strain. This can lead to large increases in toughness, as can be seen from the large failure strains achieved in self-reinforced composites [1-6], steel fibre composites [7, 8] and coir composites [9]. However, in all of these examples, the increased failure strain is achieved at the expense of a reduced stiffness or strength. This illustrates the toughness-stiffness dilemma, which states that materials are usually either tough or stiff, but seldomly both.

Fibre hybridisation is one way to break through the stiffness-toughness dilemma [10-12] by changing the balance of these two important material properties. By combining both fibre types in a suitable manner, toughness and stiffness can be achieved in a single composite material. In general, three hybrid configurations are available to the material designer: these are termed interlayer, intralayer and intrayarn hybridisation. In interlayer hybrids, each ply contains only one fibre type, but plies of different fibre types are stacked onto each other [13-16]. In intralayer hybrids, each ply contains both fibre types, for example by co-weaving [17]. In intrayarn hybrids, the fibre types are mingled within a yarn [18-21].

The interlayer configuration is the most common configuration, as it is the easiest one to produce. The intralayer configuration yields a better dispersion of the fibre types, but is more difficult to 
produce as it requires specialist weaving techniques. The intrayarn configuration has, potentially, the highest dispersion, but it is hard to find such materials in a commercial product format. The dispersion is thought to be vital, as it is generally accepted that a better dispersion improves the overall mechanical performance of hybrid composites $[10,11]$. Nevertheless, the number of direct comparisons between these configurations is limited. Pegoretti et al. [22] found that intralayer hybrids outperform interlayer hybrids for crack propagation in penetration impact tests. Park and Jang [23] found that intralayer hybrids have a smaller delaminated area after penetration compared to interlayer hybrids.

The failure strain improvements that are possible through hybridisation are limited by the failure strain of the fibres that are added. The most common hybridisation fibre for carbon fibre $[10,11]$ is glass fibre, which has a failure strain of $3-5.5 \%$. This limits the potential failure strain of carbon/glass hybrids to just $4 \%$. Large improvements are only possible when a very ductile fibre is added, for instance an oriented polymeric fibre. Hine et al. [18] hybridised carbon fibres with polyamide fibres and achieved large ductility improvements in bending, but not in tension. Similarly, Taketa et al. [13] and Swolfs et al. [17, 24] hybridised carbon fibres with self-reinforced polypropylene (SRPP). The 20\% failure strain of SRPP provides a large potential for enlarging the ultimate failure strain of the hybrid composite. Whereas Taketa et al. did not report on the ultimate failure strains, Swolfs et al. maintained an ultimate failure strain of $20 \%$ in their hybrid composites. This was achieved despite replacing half of the volume of SRPP with carbon fibre-reinforced polypropylene.

Another crucial parameter in hybrid composites is the bonding between the layers. Bunsell and Harris [25] showed that an unbonded carbon/glass hybrid composite had a vertical load drop when the carbon fibre layers broke in tension. When the layers were well bonded, the tensile behaviour changed to a more gradual transfer of the load into the glass layers. The importance of the interlayer bonding was also illustrated in the works of Czél, Jalalvand and Wisnom [16, 26, 27]. They identified the mode II fracture toughness as a key parameter controlling the damage mechanisms in carbon/glass hybrid composites.

Recently, Swolfs et al. [17] also showed that too strong bonding reduced the ultimate failure strain in intralayer carbon fibre/self-reinforced hybrid composites. This occurred when isotropic matrix films were added in between the layers during the production process. The matrix added by these films improved the bonding in hot compacted self-reinforced composites [4, 28, 29]. The strong bonding prevented the debonding of the CF prepregs over their entire length, which localised the strain in a small region. In hybrid carbon fibre/self-reinforced composites however, excess matrix in the $\mathrm{CF}$ prepregs may also contribute to an improved bonding. It is therefore likely that the bonding will depend on the carbon fibre volume fraction $\left(\mathrm{V}_{\mathrm{f}}\right)$. The goal of this study is to investigate whether this is indeed the case. If so, we will highlight how this affects the mechanical performance. Two different CF-reinforced PP prepregs with a different type of PP matrix and thereby a different level of adhesion will be used to further understand the observed differences. One of these CF prepregs used a standard PP homopolymer while the other used a maleic anhydride grafted PP.

\section{Materials and methods}

\subsection{Materials}

Drawn polypropylene (PP) tapes were provided by Propex Fabrics GmbH (Germany). These homopolymer tapes have a draw ratio of $10-15$, resulting in a tensile modulus of $10 \mathrm{GPa}$ and tensile 
strength of $500 \mathrm{MPa}$ [3]. Their thickness and width are $50 \mu \mathrm{m}$ and $2.4 \mathrm{~mm}$ respectively. A $20 \mu \mathrm{m}$ isotropic film made from the same PP grade was provided as well.

CF prepregs were sourced from Jonam Composites (UK) and Mitsuya (Japan). Their dimensions and properties are summarised in Table 1. The Jonam prepregs contained a homopolymer PP matrix, whereas the Mitsuya prepregs contained a maleic anhydride (MA) grafted PP matrix. The percentage of MA is unknown. These CF prepregs are referred to as CFPP and CFMAPP, respectively. The fibres in both prepregs have very similar mechanical properties (see Table 1). The fibre volume fractions of the CFPP and CFMAPP prepregs are $32 \% \pm 1 \%$ and $46 \% \pm 2 \%$, respectively. Their microstructure is clearly different (see Fig. 1). Fig. 1a shows resin-rich outer regions for the CFPP prepregs, which are absent for the CFMAPP prepregs in Fig. 1b.

Table 1: Comparison of the two types of prepregs.

\begin{tabular}{ccc}
\hline & CFPP prepregs & CFMAPP prepregs \\
\hline Manufacturer & Jonam Composites (UK) & Mitsuya (Japan) \\
Matrix & Homopolymer PP & MAPP \\
Melting temperature of matrix $\left({ }^{\circ} \mathrm{C}\right)$ & $163 \pm 1$ & $139 \pm 1$ \\
Dimensions $(\mathrm{mm})$ & $3 \times 0.160$ & $5 \times 0.06$ \\
Fibre type & $T 700 S$ & TR50S \\
Fibre tensile modulus $(\mathrm{GPa})$ & 230 & 240 \\
Fibre tensile strength $(\mathrm{MPa})$ & 4900 & 4900 \\
Fibre failure strain & $2.1 \%$ & $2.1 \%$ \\
Fibre volume fraction & $32 \% \pm 1 \%$ & $46 \% \pm 1 \%$ \\
\hline
\end{tabular}
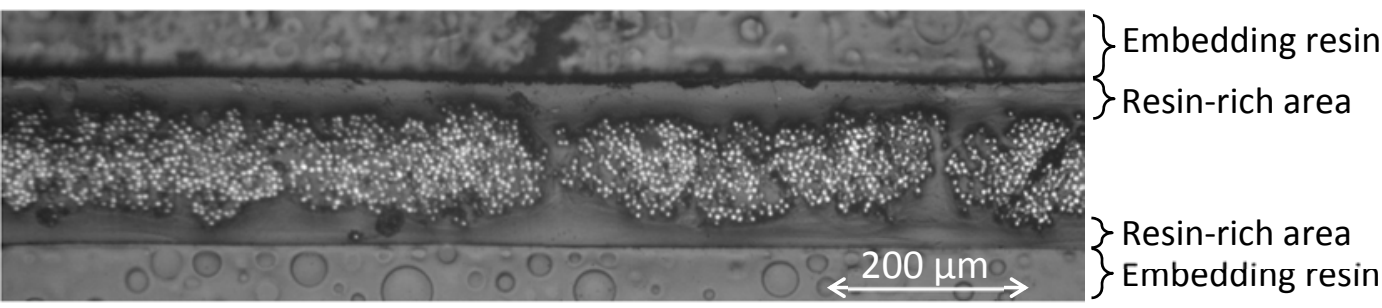

(a)

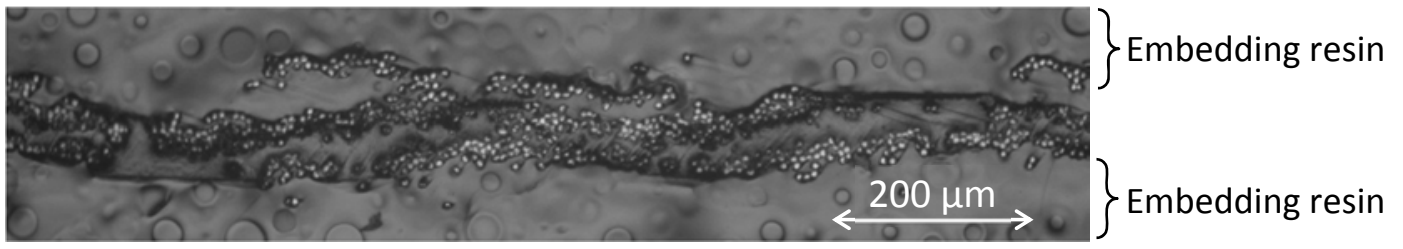

(b)

Figure 1: Microstructures of (a) CFPP prepregs, and (b) CFMAPP prepregs. The boundary between prepreg and embedding material is not always clearly visible for the CFMAPP prepregs.

The CF prepregs were co-woven with PP tapes by Propex Fabrics GmbH (Germany) in different volume fractions. All warp yarns are PP tapes, while the weft directions contain both PP tapes and CF prepregs. For the CFPP hybrids, 1/13, 1/7 and 1/3 of the PP tapes were replaced by CFPP prepregs. These hybrids will be referred to as $3 \%, 7 \%$ and $11 \%$, respectively (see Table 2 ). For the 
CFMAPP hybrids, $1 / 8$ and $1 / 3$ of the PP tapes were replaced by CFMAPP prepregs. These hybrids are referred to as $7 \% \mathrm{MA}$ and $16 \% \mathrm{MA}$, respectively (see Table 2 ).

Table 2: Carbon fibre volume fractions and sample thicknesses for all hybrids.

\begin{tabular}{cccc}
\hline Type of hybrid & Label & Carbon fibre $\mathrm{V}_{\mathrm{f}}$ & Sample thickness (mm) \\
\hline Non-hybrid & $0 \%$ & $0 \%$ & $1.23 \pm 0.02$ \\
\hline \multirow{2}{*}{ CFPP } & $3 \%$ & $3.4 \% \pm 0.1 \%$ & $1.34 \pm 0.01$ \\
& $7 \%$ & $6.9 \% \pm 0.2 \%$ & $1.47 \pm 0.02$ \\
& $11 \%$ & $11.0 \% \pm 0.4 \%$ & $1.67 \pm 0.01$ \\
\hline \multirow{2}{*}{ CFMAPP } & $7 \% \mathrm{MA}$ & $7.0 \% \pm 0.2 \%$ & $1.40+0.02$ \\
& $16 \% \mathrm{MA}$ & $15.7 \% \pm 0.6 \%$ & $1.96 \pm 0.02$ \\
\hline
\end{tabular}

The PP tapes were woven into a twill $2 / 2$ pattern, but the CF prepregs were only interlaced with this pattern every four tapes (see Fig. 2). This places the CF prepregs more towards one side of the cloth, similar to a sateen weave. If the prepreg side of the cloth is placed upwards for layers above the neutral line of the layup and downwards for layers below the neutral line, then flexural modulus can be further improved. This architecture therefore allows lay-ups with improved flexural properties compared to an architecture with the CF prepregs in the middle of the cloth. The fewer interlacing points also reduce the crimp of the CF prepregs, which should lead to better surface quality and mechanical performance.

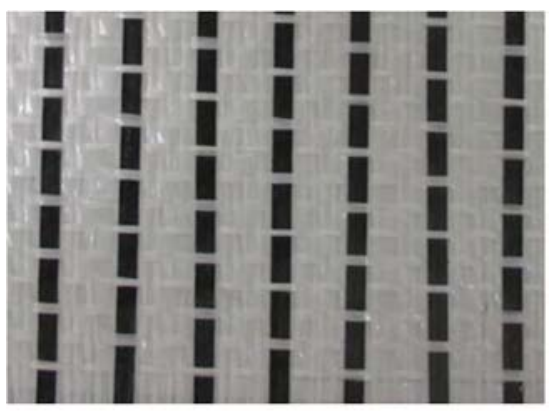

(a)

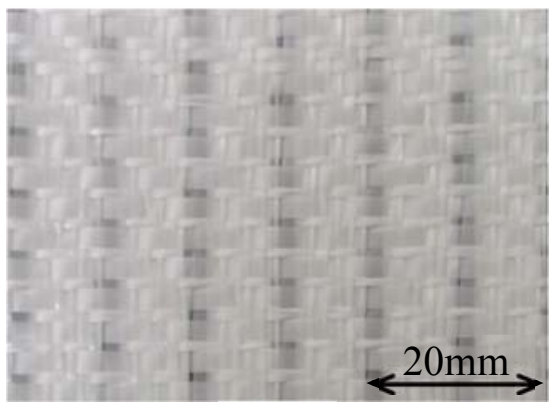

(b)

Figure 2: Co-woven CFPP hybrid cloth with a fibre volume fraction of 7\%: (a) front face, and (b) back face of the fabric.

\subsection{Hot compaction}

For tensile, flexural and impact, the hybrid cloths were stacked in a (0/90/0/90)s layup (see Fig. 3). The weft direction contains the CF prepregs, making it the stiffest and strongest direction. This 
direction is therefore labelled as the $0^{\circ}$ direction. The hybrid cloths were always oriented with the CF prepregs towards the outside. The relative position of the CF prepregs in two distinct layers was not controlled. PP films were not added in between the layers.

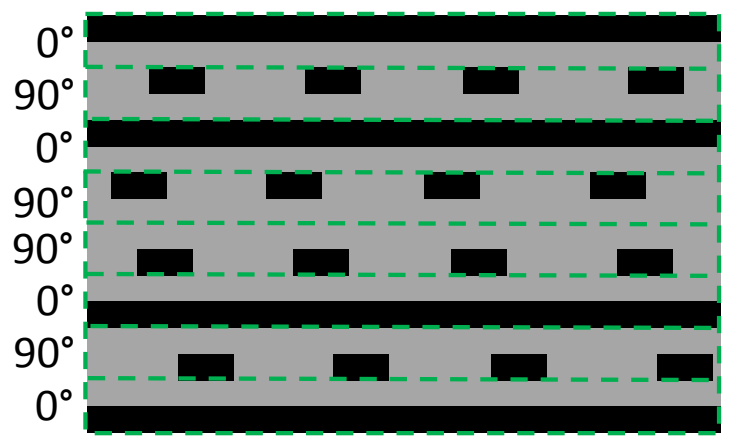

Figure 3: Schematic for the (0/90/0/90)s layup that was used for tensile, flexural and impact samples. The black regions are the carbon fibre prepregs, whereas the dashed lines indicate the boundaries of the layers.

The layup was placed in between two aluminium cover plates without any frame and inserted into a hot press at $188^{\circ} \mathrm{C}$. The press was preheated for $10 \mathrm{~min}$ prior to inserting the layup to ensure a homogeneous temperature distribution over the press platens. After the layup was inserted, a pressure of $3.9 \mathrm{MPa}$ was exerted onto the material for $5 \mathrm{~min}$. Then, the layup was cooled down to $40^{\circ} \mathrm{C}$ in about $5 \mathrm{~min}$, while maintaining the pressure.

\subsection{Peel strength tests}

To assess the bonding in these intralayer hybrids, T-peel strength tests were performed according to ASTM D1876. It should be noted that this test actually evaluates the interlayer bonding between two intralayer hybridised layers instead of the intralayer bonding. Intralayer bonding is the parameter controlling the debonding of the CF prepregs from the surrounding PP tapes. Interlayer bonding controls the delaminations on the layer level, whereas intralayer bonding controls the debonding within a layer. Since direct measurements of the intralayer bonding are highly challenging, it is hypothesised that the intralayer bonding is strongly correlated with the peel strength. This hypothesis is reasonable for this specific hybrid cloth, as the architecture has the CF prepregs mainly on one side of the fabric (see Fig. 2). The bonding between the CF prepregs and the SRPP within the same layer is therefore nearly the same as that with the SRPP of the adjacent layer. We will consistently refer to intralayer instead of interlayer bonding, as it is the key parameter determining the mechanical properties of intralayer hybrids.

It should also be remarked that peel strength tests is a mode I test, even though delamination and debonding in tension, flexure and impact is controlled by mode II. The low flexural modulus of SRPP and its hybrids unfortunately do not allow carrying out a proper fracture toughness test, such as the double cantilever beam test for mode I or the end-notch flexure test for mode II fracture toughness. The peel strength test is therefore the best alternative. Mode I and mode II in SRPP and its hybrids are likely to be controlled by the amount of matrix present in between the layers, and should therefore exhibit similar tendencies.

It is important to note that the top and bottom of the co-woven cloths are different (see Fig. 2). The weave architecture causes the CF prepregs to be preferentially on one side of the cloth. Four cowoven cloths were therefore stacked with the CF prepreg side towards the middle of the layup, 
resulting in a symmetric layup (see Fig. 4a). A $12 \mu \mathrm{m}$ polyimide peel ply between the second and third cloth was hence in direct contact with the CF prepregs. Also, the addition of CF prepregs increases the stiffness of the peel strength. For a correct peel strength test, however, the legs of the specimen should be compliant. Therefore, the peel samples had the CF prepregs perpendicular to the peeling direction (see Fig. 4b). In this case, the stiffness of the legs is nearly the same as for SRPP. The hot compaction parameters were exactly the same as for the other.

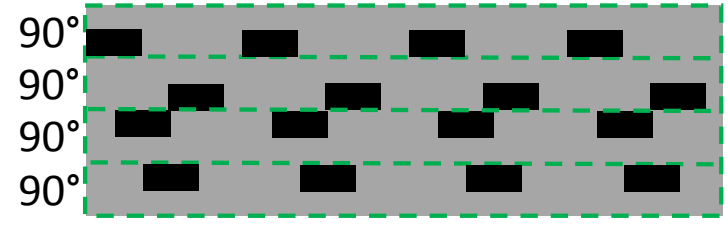

(a)

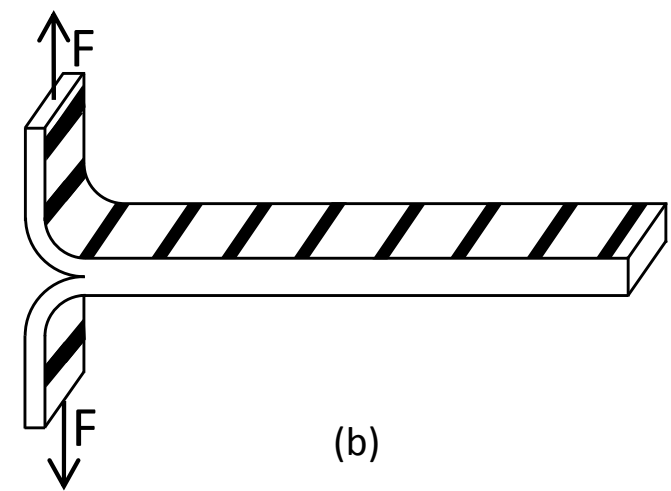

Figure 4: Peel strength samples: (a) schematic of the (90) 4 layup, and (b) the peel strength test with carbon fibre prepregs transverse to the peeling direction. The black regions are the carbon fibre prepregs, whereas the dashed lines indicate the boundaries of the layers.

The specimens were cut down to a width of $20 \mathrm{~mm}$ and a length of $300 \mathrm{~mm}$ using a sharp knife. The nominal specimen thickness was $0.6 \mathrm{~mm}$. The specimens were cut in such a way that the length of the insert film, which is equivalent to the unbonded length, was $76 \mathrm{~mm}$. The two unbonded ends were pulled apart at a rate of $254 \mathrm{~mm} / \mathrm{min}$. The specimens were tested at room temperature on an Instron 5943 tensile machine with a $1 \mathrm{kN}$ load cell. The peel strength was defined as the average peel load per mm width of the specimen. The average was calculated over the first $127 \mathrm{~mm}$ displacement after the initial load peak, as prescribed by the standard. At least 10 specimens were tested for each configuration. The specimens were tested in random order to minimise systematic errors.

\subsection{Tensile tests}

Tensile tests were performed according to ASTM D3039. Tests were conducted on an Instron 4505 tensile machine equipped with a $100 \mathrm{kN}$ load cell and hydraulic grips. Rectangular specimens of $250 \times 25 \mathrm{~mm}$ were tested at a gauge length of $150 \mathrm{~mm}$. The specimen thickness is summarised in Table 2. Sandpaper was used as end-tabs to avoid slippage in the clamps. The applied strain rate was $5 \% / \mathrm{min}$ to ensure failure in about 5 minutes, as recommended by the standard. At least 4 specimens were tested for each configuration.

A speckle pattern was applied to the surface. This speckle pattern was tracked by a camera throughout the tensile test. Digital image correlation of the images was then performed to calculate the average surface strain in the longitudinal direction. After the CF prepreg failure, the specimen surface was damaged and the surface strain could not be measured anymore. The approach described in Swolfs et al. [17, 24] was used to resolve this issue. The crosshead displacement of the tensile machine was used to calculate the strain after the carbon fibre failure. This strain was shifted by a constant factor to ensure strain continuity when the CF prepregs fail. The tensile modulus was calculated as the slope between $0.1 \%$ and $0.3 \%$ strain. 


\subsection{Flexural tests}

Three point flexural tests were performed according to the ASTM D790 standard. The span length was chosen to be $60 \mathrm{~mm}$ for all specimens. This corresponds to an average span-to-thickness ratio of 40 and a minimal ratio of 30 . The rollers had a diameter of $10 \mathrm{~mm}$. The displacement rate was 4 $\mathrm{mm} / \mathrm{min}$, corresponding to a strain rate of $1 \% / \mathrm{min}$ on average for the outer surface, as recommended by the standard. This displacement rate was applied to all specimens, irrespective of their exact thickness. Some strain rate differences between the different hybrids were hence inevitable, but we do not expect a large strain rate sensitivity in this material. The nominal specimen length was $90 \mathrm{~mm}$, while the nominal specimen width was $20 \mathrm{~mm}$ instead of the recommended 10 $\mathrm{mm}$. This was required to minimise scatter due to the large unit cell of some intralayer hybrid cloths. A $10 \mathrm{~mm}$ width would cause significant variations in the number of carbon fibre yarns in each specimen. At least five specimens were tested, all of which had the outer layers in the $0^{\circ}$ direction. The flexural modulus was calculated at flexural strains at the surface between 0.1 and $0.3 \%$.

\subsection{Penetration impact tests}

Falling weight impact tests were performed on a CEAST Fractovis 6789 machine, according to ISO 6603-2. A hemispherical striker with a $20 \mathrm{~mm}$ diameter was used. All specimens were clamped with a force of $5600 \mathrm{~N}$, which corresponds to an applied pressure of 3.5 MPa. The clamps were roughened to increase friction. Specimen sizes were 100x100 mm, which was large enough to avoid necking and wrinkling [30]. At least six specimens were tested for each hybrid configuration. The load was registered by a $20 \mathrm{kN}$ load cell in the striker tip, while the displacement was measured using a laser.

The striker was set to a height of $1 \mathrm{~m}$. The inner and outer diameter of the clamp were 40 and 60 $\mathrm{mm}$, respectively. The mass of the striker was $26.17 \mathrm{~kg}$, corresponding to a total energy of $257 \mathrm{~J}$. This was sufficient to cause penetration in all specimens. The energy absorption was calculated by integrating the area underneath the load-displacement diagram.

\subsection{Fibre volume fraction determination}

For most carbon fibre composites, the best approach for determining the $\mathrm{V}_{\mathrm{f}}$ is to use matrix digestion. However, the chemical inertness of PP hampers a complete digestion. Therefore, matrix burn-off tests were performed according to the ASTM D2584 standard. Specimens with a nominal weight of $2 \mathrm{~g}$ were prepared and weighed. The specimens were heated in a porcelain crucible until the specimen ignited. The specimens were then inserted into a muffle furnace for $2 \mathrm{~h}$ to remove the carbonaceous residue. The ASTM standard recommends a furnace temperature of $565^{\circ} \mathrm{C}$, but this was found to remove the carbon fibre. Instead, the temperature was set to $450^{\circ} \mathrm{C}$, where no further weight loss was found even after $48 \mathrm{~h}$. The $\mathrm{V}_{\mathrm{f}}$ of carbon fibre was determined based on the weight before and after burn-off. This calculation assumes densities of $1800 \mathrm{~kg} / \mathrm{m}^{3}$ and $920 \mathrm{~kg} / \mathrm{m}^{3}$ for the carbon fibre and PP/MAPP respectively.

The matrix burn-off method was used to determine the fibre $\mathrm{V}_{\mathrm{f}}$ in the CF prepregs. Unfortunately, the low $\mathrm{V}_{\mathrm{f}}$ in the hybrid composites limits the accuracy of this method. Therefore, an alternative method was set up based on the areal densities. Three rectangular hybrid cloths of at least $100 \mathrm{~cm}^{2}$ were cut and their width and length were measured. Their weight was measured to an accuracy of $0.1 \mathrm{mg}$. The CF prepregs were then carefully drawn out of the cloth, weighed separately and divided by the dimensions of the hybrid cloth. The ratio of the areal densities of the CF prepregs 
and the hybrid cloth yielded the weight fraction of CF prepregs in the hybrid composite. This fraction could be converted into the weight fraction of carbon fibre in the hybrid composite. Such calculation required the weight fraction of carbon fibre in the CF prepreg, which was accurately determined from matrix burn-off. This yielded all the necessary information to calculate the carbon fibre $\mathrm{V}_{\mathrm{f}}$ in the hybrid cloth.

\subsection{Classical laminate theory}

The stiffness of the intralayer hybrid composites was modelled using the classical laminate theory (CLT). The following assumptions were used:

- A co-woven cloth is composed of a woven SRPP and a UD CF prepreg layer. The relative fractions are determined by the $\mathrm{V}_{\mathrm{f}}$ of the $\mathrm{CF}$ prepreg.

- The CF prepregs are homogeneously distributed over the thickness of a layer, even though they are actually located more towards the outside of the lay-up.

- The crimp of the CF prepregs in the weave is neglected. The high width-to-thickness ratio of the PP tapes and CF prepregs ensures this crimp is below $0.2 \%$, according to a geometrical model built in WiseTex [31]. This assumption introduces an error in the tensile modulus of $0.5 \%$ in the worst case scenario.

For the CF prepregs, the measured fibre volume fractions were used. The $\mathrm{V}_{\mathrm{f}}$ of PP tapes in SRPP was difficult to determine experimentally because matrix and tape have similar physical properties. Optical microscopy did not provide sufficient contrast between tape and matrix. DSC has also been used to quantify tape volume fractions, but this requires clearly distinct endotherms for matrix and tape. Unfortunately, this was not the case for the particular SRPP used here. Therefore, a tape $\mathrm{V}_{\mathrm{f}}$ of $70 \%$ is assumed. This implies that $30 \%$ of the PP tapes melted during hot compaction, which is a reasonable value for SRPP [32].

Chamis' formulae were used to calculate the engineering constants of UD SRPP and UD CF prepreg layers [33]. The input parameters for these formulae are summarised in Table 3. The longitudinal modulus of carbon fibre was taken from their data sheets, whereas the other engineering constants were estimated from literature [34, 35]. The transverse tensile modulus, longitudinal shear modulus and Poisson's ratio of the PP tapes were estimated from [36].

Table 3: Engineering constants used as input for Chamis' formulae [2,30-32].

\begin{tabular}{lccc}
\hline & Carbon fibre & PP tape & PP matrix \\
\hline Longitudinal tensile modulus (GPa) & 230 & 6.9 & 1.5 \\
Transverse tensile modulus (GPa) & 15 & 1 & 1.5 \\
Longitudinal shear modulus (GPa) & 13.7 & 0.847 & 0.536 \\
Longitudinal Poisson's ratio (-) & 0.25 & 0.4 & 0.4 \\
\hline
\end{tabular}

The longitudinal modulus of the PP tapes is $10 \mathrm{GPa}$ prior to hot compaction [3]. This modulus will however decrease due to molecular relaxation during hot compaction, which implies that the actual modulus of the PP tapes in SRPP is unknown. The modulus value was hence adapted to yield the measured tensile modulus for a 0/90 lay-up of UD SRPP. This approach neglects the limited crimp in the PP tape weave, but corrects for it by changing the longitudinal tensile modulus of the PP tape. The resulting estimate of the tensile modulus after hot compaction was $6.9 \mathrm{GPa}$. This value agrees 
well with the measured tensile modulus after an annealing treatment that represents the hot compaction process [3].

The matrix tensile modulus was measured for the PP grade used in the tapes and published elsewhere [3]. The same properties were assumed for the PP matrix inside the CF prepregs, although its properties are actually unknown. This is justified by the fact that the elastic properties of the CF prepreg are anyway dominated by the elastic properties of the carbon fibres. The matrix Poisson's ratio was chosen as a typical value for PP.

\section{Experimental results}

The study focuses on tensile, flexural and impact properties. Our previous work [17] illustrated the importance of the intralayer bonding for the tensile behaviour of these hybrid composites. Therefore, this section starts off by analysing the peel strength, followed by an investigation of the tensile, flexural and impact properties.

\subsection{Peel strength}

It is well-known that the peel strength of non-hybrid SRPP increases when woven layers of PP tapes are interleaved with PP films [2, 28, 29]. Adding more CF prepregs may cause a similar increase in the intralayer bonding, as the CF prepregs add extra matrix material to the hybrid. The PP matrices in the prepregs are of an unknown grade, which may also influence the peel strength. For the CFPP prepregs, the homopolymer PP has the same melting temperature as the PP grade of the films and tapes (see Table 1), indicating that both grades are relatively similar. For the CFMAPP prepregs however, the melting temperature of $139^{\circ} \mathrm{C}$ is significantly lower due to the grafting of MAPP. The altered chemical structure of MAPP improves both the adhesion between CF and PP [37], and the bonding between CFMAPP and SRPP. As an additional effect, the MAPP also spreads out in between the layers, and does not just stay within the prepregs.

Representative peel force diagrams are shown in Fig. 5. While there is relatively little variation in the peel force for the $0 \%$ reference, this is increased by adding CFPP prepregs. The variation becomes even larger for the CFMAPP prepregs due to their better adhesion. The distance between the peaks is two times the spacing between the prepregs. The peel strength was found to be proportional to the carbon fibre $\mathrm{V}_{\mathrm{f}}$ for both the CFPP and CFMAPP hybrids (see Fig. 6). The peel strength of the CFPP hybrid with the highest $V_{f}$ was measured as nearly the same as for the nonhybrid SRPP with films. It should be noted that the observed increase is not caused by the increased stiffness of the layers. This unwanted influence was avoided by performing the peel strength tests on specimens with carbon fibres only in the $90^{\circ}$ direction. The additional carbon fibres will therefore have only a minimal effect on the stiffness of the peel samples.

The maleic anhydride in the CFMAPP hybrids caused a higher peel strength due to the stronger adhesion of MAPP to carbon fibre and PP tapes. For the same reason, the peel strength also increases faster with fibre volume fraction than for the CFPP hybrids. For the $16 \% \mathrm{MA}$ hybrid, the peel strength is significantly higher than the values for SRPP with films, which is $1 \mathrm{~N} / \mathrm{mm}$ or lower. The next sections will analyse how these differences in peel strength or intralayer bonding influence the mechanical properties of the hybrid composites. 


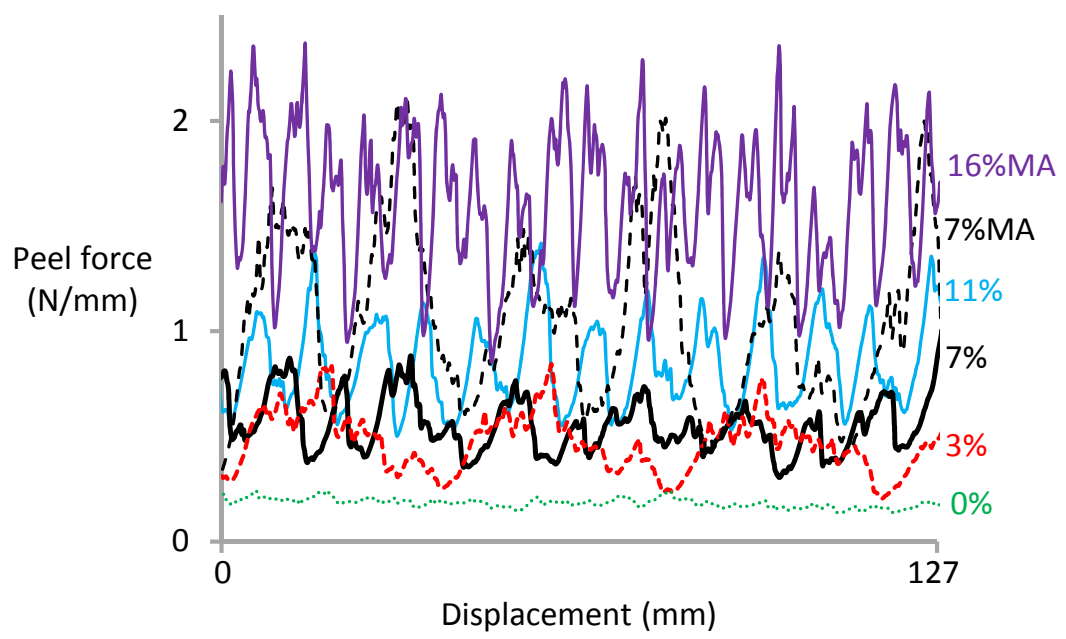

Figure 5: Representative peel force diagrams for hybrid composites with CFPP and CFMAPP prepregs.

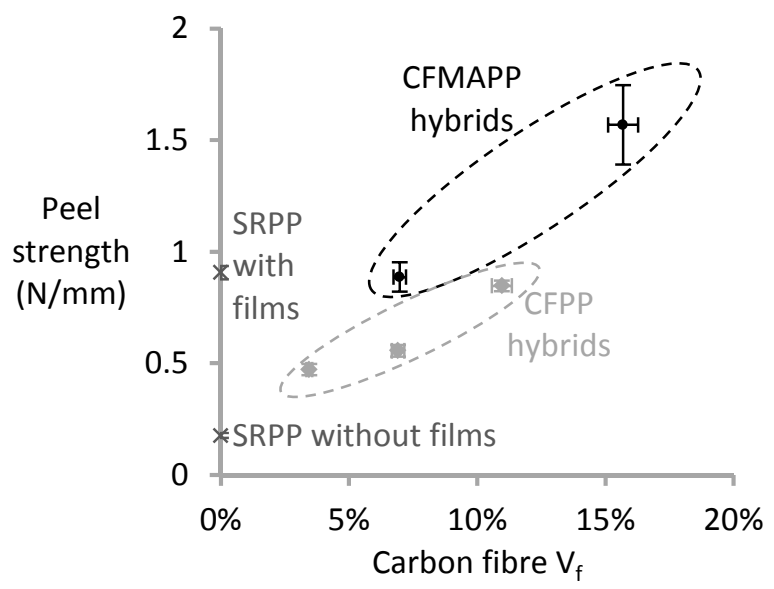

Figure 6: Peel strength of hybrid composites with CFPP and CFMAPP prepregs as a function of the carbon fibre $V_{f}$. The peel strength values for SRPP with and without PP films were added to facilitate comparison. These films were not

\subsection{Tensile properties} used in the hybrids.

In carbon fibre/self-reinforced hybrid composites, the addition of films strongly reduced the ultimate failure strain in tension [17]. This was attributed to the increased intralayer bonding. The increased peel strength due to the increased carbon fibre $V_{f}$ may therefore have a similar effect here.

Representative tensile diagrams of the hybrid composites are shown in Fig. 7. For the CFPP hybrids, the $20 \%$ ultimate failure strain of the non-hybrid SRPP is maintained up to a carbon fibre $\mathrm{V}_{\mathrm{f}}$ of $7 \%$. For the $11 \%$ hybrid however, the ultimate failure strain is reduced to around $6 \%$. This reduction occurs faster for the well-adhered CFMAPP hybrids.

It should be noted that Swolfs et al. [17] maintained a high ultimate failure strain, even at a CF volume fraction of $22 \%$. This was achieved for similar hybrid composites, where the main difference was the CF prepregs. The CF volume fraction in their CFPP prepregs was $47 \%$, compared to $32 \%$ in this study. It is therefore expected that the increase in bonding strength with increased CF prepreg fraction (see Fig. 6) was more pronounced in the present study than in Swolfs et al. [17]. A further reason may be the resin-rich zones on the top and bottom of the CFPP prepregs 
in this study (see Fig. 1a), which act as an additional matrix film. This matrix film also originates from a different PP grade than the PP tapes, and hence has different mechanical properties.

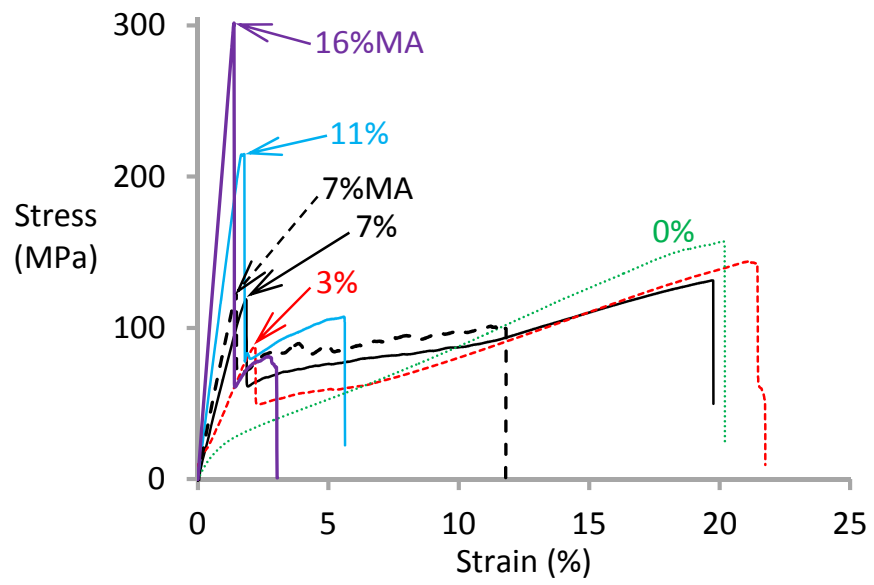

Figure 7: Representative tensile diagrams of the hybrid composites for different carbon fibre $V_{f}$.

In the 7\%MA hybrids, the strong intralayer bonding causes a significant loss in the SRPP ductility. This loss is even more pronounced in the $16 \% \mathrm{MA}$ hybrids. This correlates with the increased peel strength in Fig. 6. For high peel strengths, the bonding within the layers is too strong, which prevents the debonding along the CF prepregs from growing. This debonding is necessary to prevent localisation of the strains in the debonded region, which would cause premature failure. Visual inspection after the tensile tests revealed that regions in between the longitudinal carbon fibre prepregs are still bonded together. This confirms that the tensile performance is determined by debonding of the carbon fibre prepregs and not by delamination. The parameter controlling the ultimate failure strain of these hybrids is hence the intralayer instead of interlayer bonding, even though both parameters are strongly correlated.

A strong intralayer bonding localises the strain in a small region of the specimen. This is illustrated in Fig. 8, which shows a specimen of the $7 \%$ and $11 \%$ hybrid after a tensile test. The $7 \%$ hybrid debonded over its entire length, whereas the $11 \%$ hybrid debonded only over a portion of its length. This strong correlation between peel strength and ultimate failure strain is confirmed in Fig. 9. For maintaining a high ultimate failure strain, the peel strength should be below $0.6 \mathrm{~N} / \mathrm{mm}$.

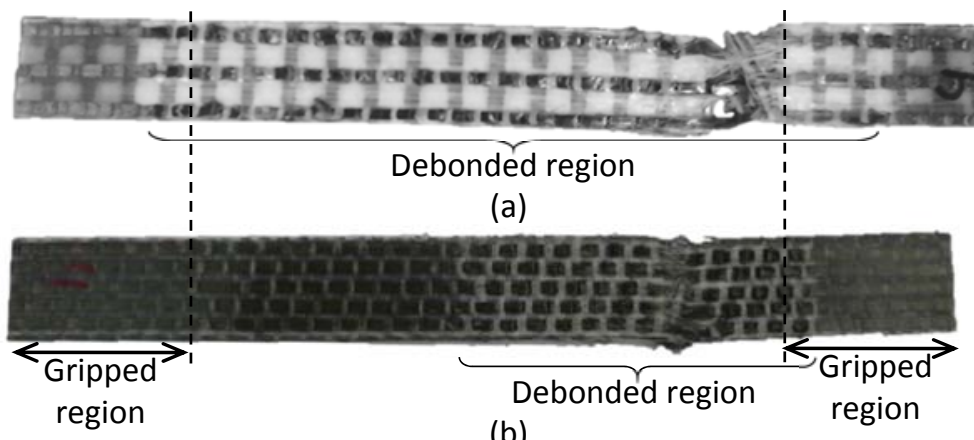

Figure 8: CFPP hybrid samples after a tensile test: (a) 7\% hybrid, and (b) 11\% hybrid. The debonded region extends slightly into the gripped region. 


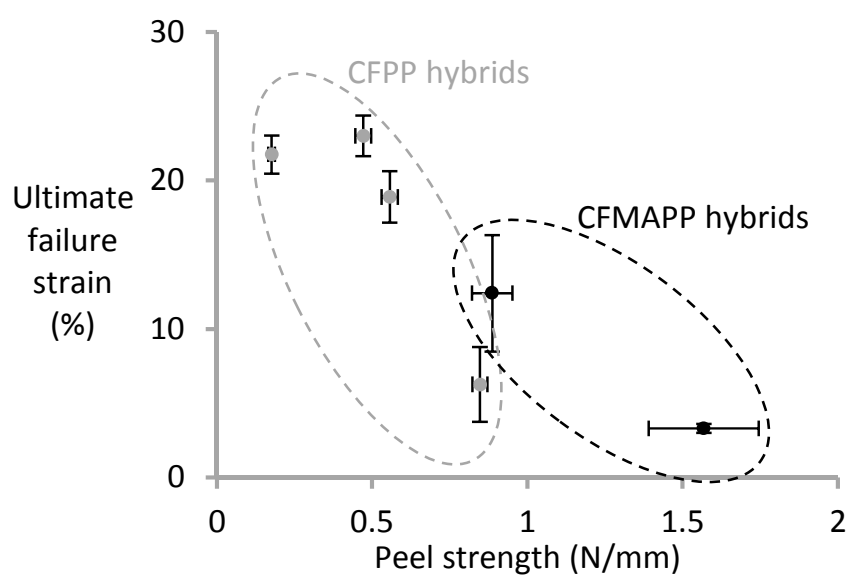

Figure 9: Correlation between peel strength and ultimate failure strain in tension.

The height of the SRPP peak in Fig. 7 depends on the fraction of SRPP in the hybrid composite. Even if this is taken into account, then the SRPP peak in the CFMAPP hybrids is still lower than in the CFPP hybrids. This indicates that some damage was inflicted to the SRPP when the CF prepregs failed. We hypothesise that the stronger adhesion between MAPP and carbon fibre localises this damage in a small region. The weaker adhesion between PP/SRRP on one hand and carbon fibre/CFPP on the other hand allows the damage to spread more along the fibres in the CFPP hybrids. This spread out damage is less detrimental for the SRPP strength, which reflects in a higher SRPP peak in Fig. 7.

Further insights can be gained by comparing the tensile moduli with the classical laminate theory (CLT) predictions (see Fig. 10). The CFPP hybrids have a significantly lower tensile modulus than the dashed line for CLT predictions. This is attributed to the shrinkage of the PP tapes during hot compaction, which creates compressive forces in the CF prepregs that cause out-of-plane undulations. These undulations have the same effect as in-plane fibre misalignment, and reduce the contribution of the carbon fibres to the stress transfer. They were less pronounced in the $11 \% \mathrm{CFPP}$ hybrid. The larger CF volume fraction provides more stability and the lower SRPP fraction reduces the shrinkage forces. This is confirmed by the tensile modulus of this hybrid being closer to the predicted values than for the 3\% and 7\% CFPP hybrids. The accuracy of the predictions is better for the CFMAPP hybrids. This is in agreement with the observation of out-of-plane undulations in the CFPP hybrids, but which were absent in the CFMAPP hybrids.

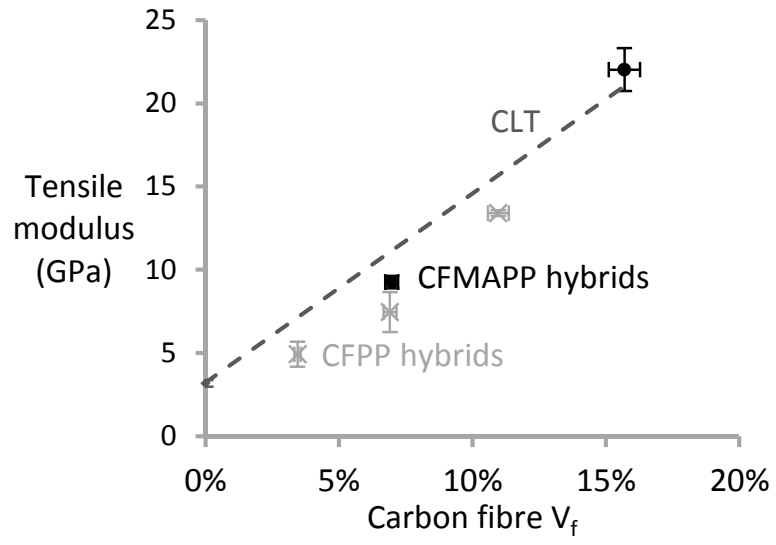

Figure 10: Tensile modulus of hybrid composites as a function of carbon fibre $V_{f}$. 


\subsection{Flexural properties}

The flexural behaviour of the hybrid composites strongly depends on the matrix type in the CF prepregs (see Fig. 11). Increasing the carbon fibre $V_{f}$ to $7 \%$ has only a small effect on the flexural behaviour for the CFPP hybrids. In contrast, the 7\%MA layup shows a much stronger increase in modulus and strength, even though the carbon fibre $\mathrm{V}_{\mathrm{f}}$ is nearly the same. Large changes in the flexural behaviour of the CFPP hybrids only occur when the carbon fibre Vf is increased to $11 \%$. Compared to the low flexural modulus of the $3 \%$ and $7 \%$, the flexural modulus of the $11 \%$ layup is three times higher. This $11 \%$ layup has a high peel strength, similar to that of the $7 \%$ MA hybrids (see Fig. 6).

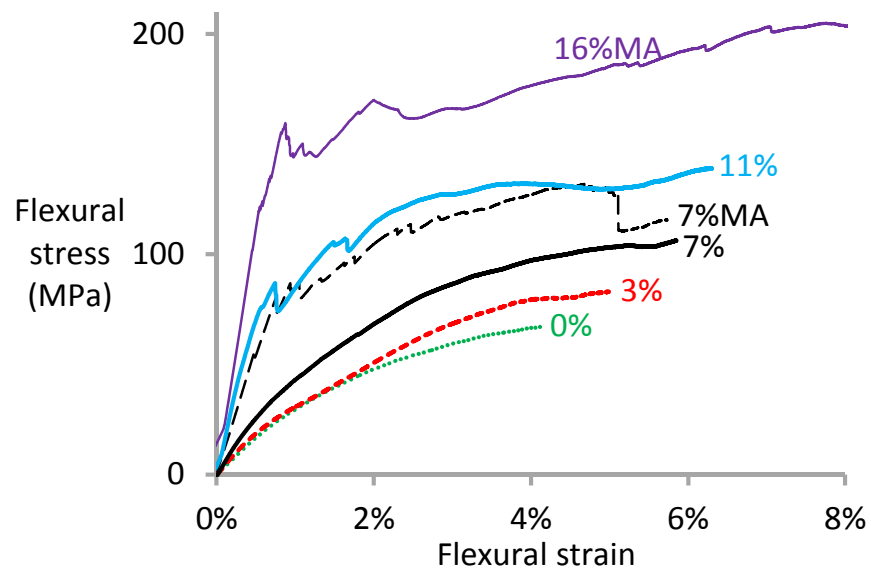

Figure 11: Flexural diagrams for the hybrid composites.

More insight can be gained when the flexural modulus is compared to CLT predictions (see Fig. 12). The measured flexural modulus is significantly lower than the predictions. This is especially true for the CFPP hybrids with $3 \%$ and $7 \%$ of carbon fibre, where the modulus increase compared to SRPP is small. The modulus increase is more pronounced for the CFMAPP hybrids. The differences between predicted and measured flexural moduli in Fig. 12 are larger than those for tensile modulus in Fig. 10. This is attributed to the compressive behaviour of the CF prepregs in flexure being more sensitive to the presence of undulations than in tension. Whereas pre-buckled fibres can unbuckle in tension, they will buckle even further in compression. It was experimentally observed that this buckling in compression occurs within the strain interval for the flexural modulus calculations. This is attributed to the low adhesion of carbon fibre to PP. CFMAPP hybrids, where the carbon fibres are not undulated, therefore use carbon fibres in a more efficient way than CFPP hybrids. 


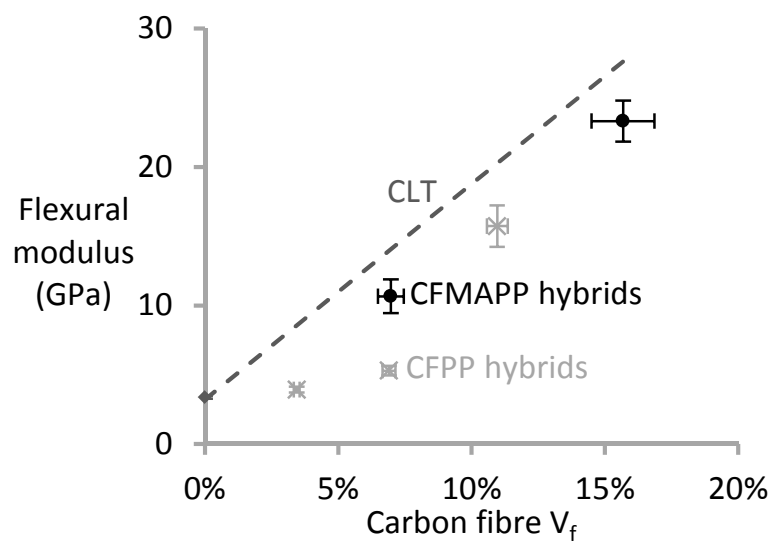

Figure 12: Flexural modulus for the hybrid composites, compared with the predictions from the classical laminate theory.

The flexural strength was not analysed, as an objective definition of this strength was difficult from the flexural diagrams in Fig. 11. The strength clearly increases by adding carbon fibres, but this increase is rather limited. This is related to the failure mechanism, which was always buckling underneath the loading nose. Increasing the roller diameters or using 4-point bending did not resolve this issue.

\subsection{Impact resistance}

The major advantage of SRPP is its impact resistance [1]. The addition of a brittle phase, such as carbon fibre, is expected to reduce the impact resistance. Nevertheless, this reduction should be minimised by an intelligent design of the hybrid composite. For facilitating the comparison, the penetration impact resistance will be compared against the linear rule-of-mixtures, as suggested by Swolfs et al. [10]. While many confounding parameters exist in impact loading, this rule-ofmixtures provides a useful framework to compare against. For the SRPP reference composite, a penetration impact resistance of $32 \pm 3 \mathrm{~J} / \mathrm{mm}$ was measured. For the all-carbon fibre reference composite, a reasonable estimate was made based on literature. Unfortunately, specific data for the penetration impact resistance of CFPP and CFMAPP was not found for similar testing conditions. Based on values for other thermoplastic carbon fibre composites, such as polyether ether ketone (PEEK) and polyphenylene sulphide (PPS) carbon fibre composites [38], an order of magnitude of $10 \mathrm{~J} / \mathrm{mm}$ seems realistic for this specific material and testing setup. This value is assumed for CFPP and CFMAPP, irrespective of the matrix and CF volume fraction.

The penetration impact resistance of the CFPP hybrids reduces by the addition of carbon fibre, but this reduction is limited (see Fig. 13). The linear rule-of-mixtures yields reasonable predictions in this case. The penetration impact resistance of the CFMAPP hybrids, however, is much poorer than for the CFPP hybrids. The linear rule-of-mixtures strongly overpredicts the measured values for the CFMAPP hybrids. The strong bonding of the MAPP makes the composite fail in a brittle manner, by limiting the extent of the debonding, delamination and PP tape fibrillation, thereby creating a local fracture (see Fig. 14d and e). The fracture region is localised along the lines of a '+'-shape, indicating a rather brittle fracture. The damage in the four lips of the '+'-shaped fracture still have a high residual stiffness, indicating that they were not debonded or delaminated. In CFPP hybrids however, the weak bonding of the PP allows debonding and delaminations (see Fig. 14a-c). This causes a fibrillated appearance and a large portion of the specimen to absorb energy. The protruding parts of the penetrated specimens feel compliant, indicating that they were debonded and 
delaminated. This indicates that a large volume of material contributed to energy absorption during impact.

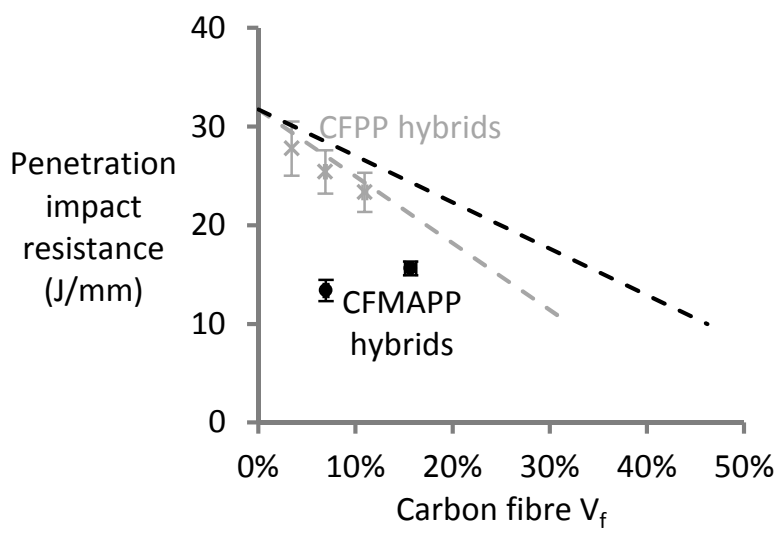

Figure 13: Penetration impact resistance for the hybrid composites, compared to the linear rule-of-mixtures.

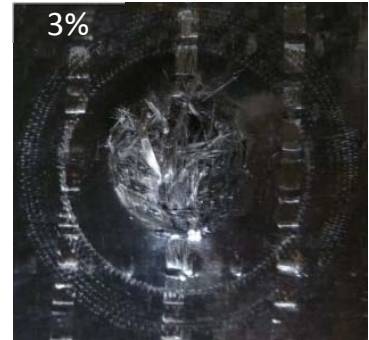

(a)

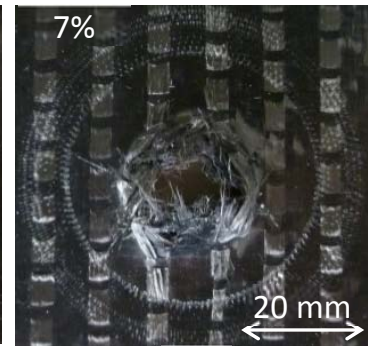

(b)

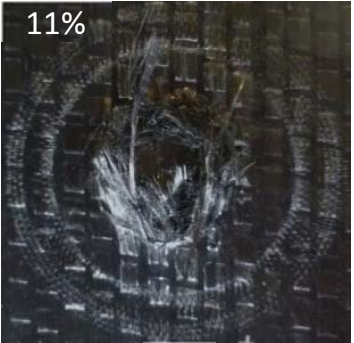

(c)

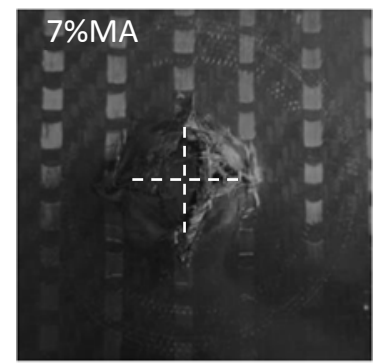

(d)

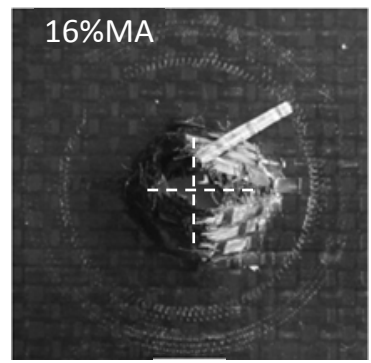

(e)

Figure 14: Sample appearance after penetration: (a) 3\%, (b) 7\%, (c) 11\%, (d) 7\%MA, and (e) 16\%MA hybrid. The CFMAPP hybrids show a '+'-shaped fracture. This feature is indicated by dashed, white lines, as it may be difficult to recognise.

More insight can be obtained from the force-displacement diagrams (see Fig. 15). It should be noted though that the thickness was not the same for all the samples (see Table 2), which makes the absolute values here less relevant. Therefore, the focus is on the general appearance of the diagrams. With the addition of more $\mathrm{CF}$, the peak force reduces and shifts to smaller displacements. This means that fracture occurs at smaller deflections. The general appearance of the diagrams however also changes. While the $0 \%$ and $3 \%$ configurations absorb only $15-20 \%$ of the energy after the peak force, this fraction is increased up to $50 \%$ for larger carbon fibre fractions. This feature is even more pronounced in the CFMAPP hybrids, where $60-65 \%$ of the energy is absorbed after the peak force. The $11 \%$ and $7 \% \mathrm{MA}$ behave similarly, which corresponds to them having a similar peel strength (see Fig. 6). 


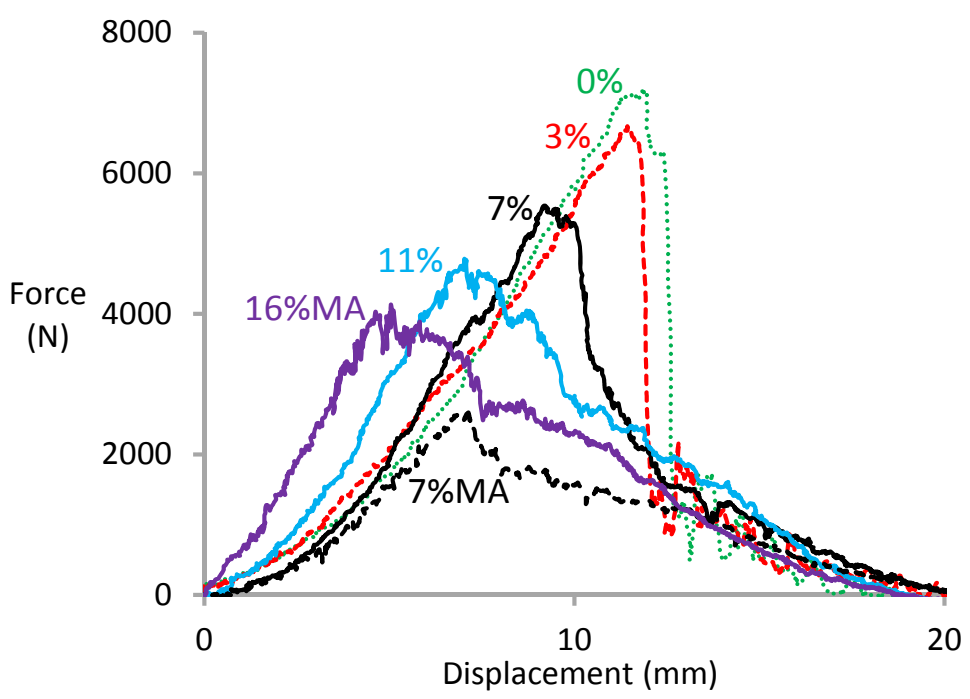

Figure 15: Representative force-displacement diagrams for the penetrating impact tests.

\section{Conclusion}

In this study, we have shown that intralayer carbon fibre/self-reinforced hybrid composites have the potential to offer a unique combination of stiffness and ultimate failure strain combined with high penetration impact resistance. Their mechanical properties were evaluated and related to the intralayer bonding. The intralayer bonding, interpreted by the peel strength test, increased with increasing carbon fibre $\mathrm{V}_{\text {f. }}$. This bonding had a significant influence on the mechanical properties of the hybrids. For a carbon fibre $\mathrm{V}_{\mathrm{f}}$ of $11 \%$, the ultimate tensile failure strain of the CFPP hybrids significantly reduced, while it was about $20 \%$ for lower $V_{f}$. The undulations caused by PP tape shrinkage resulted in relatively low flexural performance. The penetration impact resistance, however, remained high for the CFPP hybrids, and was reduced according to a linear rule-ofmixtures. This illustrates the potential of fibre hybridisation as a methodology to increase stiffness and maintain failure strain in a single composite material. This only works up to a certain fraction of the stiffer fibre, after which the failure strain does reduce.

For hybrid composites with CFMAPP prepregs, similar but more pronounced effects were observed. The intralayer bonding increased more rapidly with the addition of carbon fibres. This caused a stronger reduction in the ultimate failure strain in tension, but also led to an increased flexural modulus due to reduced undulations. The penetration impact resistance was however strongly reduced, because delaminations and debonding were hindered.

These results illustrate the importance of controlling the interfacial properties in hybrid composites. The next steps in this research will be to tune the maleic anhydride content of the CFMAPP. This should lead to an optimal balance in tensile, flexural and impact properties.

\section{Acknowledgements}

The work leading to this publication has received funding from the European Union Seventh Framework Programme (FP7/2007-2013) under the topic NMP-2009-2.5-1, as part of the project HIVOCOMP (Grant Agreement No. 246389). The authors thank the Agency for Innovation by Science and Technology in Flanders (IWT) for the grant of Y. Swolfs. I. Verpoest holds the Toray Chair in Composite Materials at KU Leuven. 


\section{References}

[1] Ward IM, Hine PJ. The science and technology of hot compaction. Polymer. 2004;45(5):141327.

[2] Swolfs Y, Zhang Q, Baets J, Verpoest I. The influence of process parameters on the properties of hot compacted self-reinforced polypropylene composites. Composites Part A: Applied Science and Manufacturing. 2014;65:38-46.

[3] Swolfs Y, Van den fonteyne W, Baets J, Verpoest I. Failure behaviour of self-reinforced polypropylene at and below room temperature. Composites Part A: Applied Science and Manufacturing. 2014;65:100-7.

[4] Swolfs Y, Crauwels L, Gorbatikh L, Verpoest I. The influence of weave architecture on the mechanical properties of self-reinforced polypropylene. Composites Part A: Applied Science and Manufacturing. 2013;53:129-36.

[5] Alcock B, Peijs T. Technology and development of self-reinforced polymer composites. In: Abe A, Kausch HH, Moller M, Pasch H, editors. Polymer composites - polyolefin fractionation polymeric peptidomimetics - collagens. Berlin: Springer-Verlag; 2013. p. 1-76.

[6] Alcock B, Cabrera N, Barkoula N, Spoelstra A, Loos J, Peijs T. The mechanical properties of woven tape all-polypropylene composites. Composites Part A: Applied Science and Manufacturing. 2007;38(1):147-61.

[7] Callens MG, Gorbatikh L, Verpoest I. Ductile steel fibre composites with brittle and ductile matrices. Composites Part A: Applied Science and Manufacturing. 2014;61:235-44.

[8] Callens MG, Gorbatikh L, Bertels E, Goderis B, Smet M, Verpoest I. Tensile behaviour of steel fibre/epoxy composites with modified adhesion. Composites Part A: Applied Science and Manufacturing. 2015;69:208-18.

[9] Defoirdt N, Biswas S, Vriese LD, Tran LQN, Acker JV, Ahsan Q, et al. Assessment of the tensile properties of coir, bamboo and jute fibre. Composites Part A: Applied Science and Manufacturing. 2010;41(5):588-95.

[10] Swolfs Y, Gorbatikh L, Verpoest I. Fibre hybridisation in polymer composites: a review. Composites Part A: Applied Science and Manufacturing. 2014;67:181-200.

[11] Kretsis G. A review of the tensile, compressive, flexural and shear properties of hybrid fibrereinforced plastics. Composites. 1987;18(1):13-23.

[12] Verpoest I, Lomov S, Swolfs Y, Jacquet P, Michaud V, Manson J-A, et al. Advanced materials enabling high-volume road transport applications of lightweight structural composite parts. Sampe J. 2014;50(3):30-7.

[13] Taketa I, Ustarroz J, Gorbatikh L, Lomov SV, Verpoest I. Interply hybrid composites with carbon fiber reinforced polypropylene and self-reinforced polypropylene. Composites Part A: Applied Science and Manufacturing. 2010;41(8):927-32.

[14] Fiore V, Valenza A, Di Bella G. Mechanical behavior of carbon/flax hybrid composites for structural applications. Journal of Composite Materials. 2012;46(17):2089-96.

[15] Sarasini F, Tirillò J, Valente M, Valente T, Cioffi S, Iannace S, et al. Effect of Basalt Fibre Hybridization on The Impact Behaviour Under Low Impact Velocity of Glass/Basalt Woven Fabric/Epoxy Resin Composites. Composites Part A: Applied Science and Manufacturing. 2013;47:109-23.

[16] Jalalvand M, Czél G, Wisnom MR. Damage analysis of pseudo-ductile thin-ply UD hybrid composites - a new analytical method. Composites Part A: Applied Science and Manufacturing, Available online. 2015;69:83-93.

[17] Swolfs Y, Crauwels L, Van Breda E, Gorbatikh L, Hine P, Ward I, et al. Tensile behaviour of intralayer hybrid composites of carbon fibre and self-reinforced polypropylene. Composites Part A: Applied Science and Manufacturing. 2014;59:78-84. 
[18] Hine PJ, Bonner M, Ward IM, Swolfs Y, Verpoest I, Mierzwa A. Hybrid carbon fibre/nylon 12 single polymer composites. Composites Part A: Applied Science and Manufacturing. 2014;65:1926.

[19] Swolfs Y, McMeeking RM, Gorbatikh L, Verpoest I. The effect of fibre dispersion on initial failure strain and cluster development in unidirectional carbon/glass hybrid composites. Composites Part A: Applied Science and Manufacturing. 2015;69:279-87.

[20] Yu H, Longana ML, Jalalvand M, Wisnom MR, Potter KD. Pseudo-ductility in intermingled carbon/glass hybrid composites with highly aligned discontinuous fibres. Composites Part A: Applied Science and Manufacturing. 2015;73(0):35-44.

[21] Swolfs Y, Gorbatikh L, Verpoest I. Stress concentrations in hybrid unidirectional fibrereinforced composites with random fibre packings. Composites Science and Technology. 2013;85:10-6.

[22] Pegoretti A, Fabbri E, Migliaresi C, Pilati F. Intraply and interply hybrid composites based on E-glass and poly(vinyl alcohol) woven fabrics: tensile and impact properties. Polymer International. 2004;53(9):1290-7.

[23] Park R, Jang J. The effects of Hybridization on the mechanical performance of aramid/polyethylene intraply fabric composites. Composites Science and Technology. 1998;58(10):1621-8.

[24] Swolfs Y, Meerten Y, Hine PJ, Ward IM, Verpoest I, Gorbatikh L. Introducing ductility in hybrid carbon fibre/self-reinforced composites through control of the damage mechanisms. Composite Structures. 2015;131:259-65.

[25] Bunsell AR, Harris B. Hybrid carbon and glass fibre composites. Composites. 1974;5(4):15764.

[26] Czél G, Wisnom MR. Demonstration of pseudo-ductility in high performance glass/epoxy composites by hybridisation with thin-ply carbon prepreg. Composites Part A: Applied Science and Manufacturing. 2013;52:23-30.

[27] Jalalvand M, Czél G, Wisnom MR. Numerical modelling of the damage modes in UD thin carbon/glass hybrid laminates. Composites Science and Technology. 2014;94:39-47.

[28] Hine P, Olley R, Ward I. The use of interleaved films for optimising the production and properties of hot compacted, self reinforced polymer composites. Composites Science and Technology. 2008;68(6):1413-21.

[29] Hine PJ, Unwin AP, Ward IM. The use of an interleaved film for optimising the properties of hot compacted polyethylene single polymer composites. Polymer. 2011;52(13):2891-8.

[30] Meerten Y, Swolfs Y, Baets J, Gorbatikh L, Verpoest I. Penetration impact testing of selfreinforced composites. Composites Part A: Applied Science and Manufacturing. 2014;68:289-95.

[31] Verpoest I, Lomov SV. Virtual textile composites software WiseTex: Integration with micromechanical, permeability and structural analysis. Composites Science and Technology. 2005;65(15-16):2563-74.

[32] Hine PJ, Ward IM, El Matty MIA, Olley RH, Bassett DC. The hot compaction of 2dimensional woven melt spun high modulus polyethylene fibres. Journal of Materials Science. 2000;35(20):5091-9.

[33] Chamis CC. Simplified Composite Micromechanics Equations for Hygral, Thermal and Mechanical Properties. NASA Technical Memorandum 88320. Houston, United States: NASA; 1983.

[34] Searles K, Odegard G, Kumosa M. Micro- and mesomechanics of 8-harness satin woven fabric composites: I - evaluation of elastic behavior. Composites Part A-Applied Science and Manufacturing. 2001;32(11):1627-55.

[35] Shindo A. Polyacrylonitrile (PAN)-based carbon fibers. In: Kelly A, Zweben C, Chou TW, editors. Comprehensive composite materials. Amsterdam: Elsevier; 2000. p. 1-33. 
[36] Ward IM, Sweeney J. Mechanical properties of solid polymers. 3rd ed. Chichester, United Kingdom: John Wiley \& Sons; 2012.

[37] Karsli NG, Aytac A. Effects of maleated polypropylene on the morphology, thermal and mechanical properties of short carbon fiber reinforced polypropylene composites. Materials \& Design. 2011;32(7):4069-73.

[38] Ghaseminejhad MN, Parvizi-Majidi A. Impact behaviour and damage tolerance of woven carbon fibre-reinforced thermoplastic composites. Composites. 1990;21(2):155-68. 